Man and Nature

L'homme et la nature

\title{
Libertinage, folie, utopie, liberté : les Amours du Chevalier de Faublas
}

\section{Pierre Gobin}

Volume 8, 1989

URI : https://id.erudit.org/iderudit/1012596ar

DOI : https://doi.org/10.7202/1012596ar

Aller au sommaire du numéro

Éditeur(s)

Canadian Society for Eighteenth-Century Studies / Société canadienne d'étude du dix-huitième siècle

ISSN

0824-3298 (imprimé)

1927-8810 (numérique)

Découvrir la revue

Citer cet article

Gobin, P. (1989). Libertinage, folie, utopie, liberté : les Amours du Chevalier de Faublas. Man and Nature / L'homme et la nature, 8, 49-61.

https://doi.org/10.7202/1012596ar

Copyright (c) Canadian Society for Eighteenth-Century Studies / Sociéte canadienne d'étude du dix-huitième siècle, 1989
Ce document est protégé par la loi sur le droit d'auteur. L'utilisation des services d'Érudit (y compris la reproduction) est assujettie à sa politique d'utilisation que vous pouvez consulter en ligne.

https://apropos.erudit.org/fr/usagers/politique-dutilisation/ 


\section{Libertinage, folie, utopie, liberté: les Amours du Chevalier de Faublas}

Le thème général du colloque de cette année semble choisi comme à, dessein pour commémorer le bicentenaire d'une oeuvre considérable, tant par son ampleur ${ }^{1}$ que par sa notoriété. En fait, l'anniversaire ne semble pas susciter d'échos et l'ensemble romanesque publié par Louvet entre 1787 et 1790, s'il est célèbre, demeure méconnu. Peu de critiques l'ont examiné autrement qu'en passant, et aucun à ma connaissance n'a accordé une grande place aux rapports originaux de la liberté avec le libertinage qui s'y trouvent exprimés. La plupart des lecteurs, encouragés en cela par le format des rééditions qui privilégient les deux premiers ouvrages qui le constituent (Une année et Six semaines) aux dépens du troisième ( $\mathrm{La}$ Fin des Amours), le considèrent comme un aimable 'livre du second rayon.' Surtout on n'a jamais accordé $\mathrm{qu}^{\prime}$ une attention distraite au fait que le romancier enrichi et 'anobli' par le succès de ses livres avait ensuite fait preuve durant la Révolution d'une grande activité politique, ${ }^{2}$ député du Loiret à la Convention, rédacteur du Journal des Débats, et, décrété d'accusation avec ses amis Girondins le 31 mai 1793, passant plus d'un an dans la clandestinité avant de revenir à Paris après la chute de Robespierre pour tenter en vain de galvaniser les vertus républicaines des Thermidoriens. Or la carrière du citoyen Louvet semble - mutatis mutandis - reprendre celle de son héros. L'écrivain, d'ailleurs conscient des affinités entre sa vie et son oeuvre, puisqu'il donna à la femme de sa vie le surnom de Lodoïska, l'une de ses héroïnes, dut, comme le chevalier, payer son 'libertinage' d'une impuissance à se faire prendre entièrement au sérieux lorsqu'il s'agissait de défendre la liberté. Nous avons en quelque sorte deux avatars structuralement voisins de la 'carrière du libertin,' non pas sanctionnés par la morale religieuse ou sociale comme c'était le cas plus tôt dans le siècle, dans la tradition illustrée par le Rake's Progress, mais inscrits dans les rapports entre les 'amours' et les 'devoirs,' la poursuite du plaisir privé et la recherche de la félicité publique. La difficulté de traduire le projet américain 'Life, liberty and the pursuit of happiness' dans une société marquée de péchés inégalitaires est vécue par le chevalier de Faublas mûri avant de l'être par son créateur, citoyen représentant du peuple. L'apparente malédiction qui pèse sur le projet politique et la carrière privée des Girondins semblent illustrées de façon exemplaire ${ }^{3}$ par l'expérience de Louvet, qui, 
toutefois, exceptionnellement, survécut à ses amis guillotinés. Mais elles sont aussi à bien des égards préfigurées par la fin des aventures de Faublas, survivant désabusé au terme du roman, et inscrites, comme en abîme, dans un récit enchâssé au début même de la suite romanesque et situé dans la malheureuse 'République' de Pologne. Si, comme le pense Aulard, Louvet était victime de ses propres fictions lorsqu'il dénonçait le 'monarchisme' de Robespierre ${ }^{4}$ il semble avoir aussi fantasmé à l'avance le déroulement de sa propre vie. Il apparaît alors comme une étrange figure de Cassandre. Sa frustration et son désespoir ne se traduiront pas par la folie comme pour Faublas, mais par une immense amertume: ${ }^{5}$ comme son personnage, l'écrivain porte le deuil de sa passion pour le bonheur.

Une lecture croisée de la fiction et de la vie me semble donc éclairer non seulement le drame du 'libertinage léger,' mais aussi celui de la 'liberté fraîche et joyeuse' qui se recoupent entre 1787 et 1795, autour de l'année-charnière de 1790 qui semblait manifester l'utopie réalisée grâce à la Révolution. Le roman, comme l'histoire qui lui succède, commence le mieux du monde: tout semble réussir au jeune Faublas imberbe, espèce de Chérubin ${ }^{6}$ que le beau sexe accueille avec faveur, éduque, protège, excuse, et que les hommes traitent avec amusement ou indulgence, quand ils ne sont pas dupes de ses travestissements et de ses intrigues; comme son charmant personnage, le jeune romancier est choyé, aimé, admiré, écouté, et réussit dans tout ce qu'il entreprend. Mais quand il touche au triomphe et au bonheur stable (il va épouser Sophie, âme-soeur révélée en outre comme Dorlinska, l'héritière de l'héroïque Lödoïska) le destin de Faublas est traversé (Six Semaines): enlèvement de la fiancée, embastillement du héros, révélations compromettantes; La Fin des Amours voit les persécutions s'accumuler, et le protagoniste est comme ligoté par les intrigues jadis ourdies inconsidérément, jusqu'au point où, tentant de trancher, il provoque la mort de ses deux plus tendres amies et sombre dans une folie dont seule l'intervention de Sophie pourra le tirer - au prix de l'exil; Louvet, lui, champion des Girondins, 'David devant le Goliath Robespierre' voit aussi le triomphe lui échapper, les incompréhensions se multiplier (avec les Jacobins qui l'excluent, avec les Girondins qui ne le suivent pas), le pouvoir le pourchasser (décrété d'accusation le 2 juin 93, fuyard associé malgré lui à l'équipée de Wimpfen, contraint sans doute à se terrer en Suisse), et malgré son 'retour en grâce' demeure la proie du malheur, malgré le dévouement et la fidélité de Lodoïska. ${ }^{7}$ Dans la fiction, comme dans l'histoire, une profonde ironie intervient; de façon évidente dans les deux cas, 'la Roche Tarpéienne est près du Capitole,' au point que les premières pages de Six Semaines ont quelque chose de mélodramatique 
Ma bouche répétait à Sophie un serment qu'avouait mon coeur, lorsque la voûte sacrée retentit d'un cri lamentable et perçant (p. 720);

dans les deux cas, le protagoniste d'ordinaire disert, prompt à la décision, se trouve démuni devant la crise:

Une obscurité profonde couvre les desseins de nos ennemis, une incertitude affreuse enchaîne toutes mes facultés

rapporte Faublas (Six Semaines, pp. 722-23) alors que l'éloquence du député à la Convention semble inopérante; dans les deux cas ce qui justifiait l'action se trouve arraché, d'où un immense vide dans l'esprit du héros, une béance que viennent emplir des fantasmes effrayants; dans les deux cas enfin et surtout, la consécration entrevue, qui devait marquer le vrai bonheur, la vraie liberté, par l'accord 'de la bouche et du coeur,' se trouve écartée à l'instant même où le héros s'en trouve digne; on voit brisé ce qui devait sanctionner l'accès à la maturité véritable, la prise de responsabilité, la découverte des véritables lumières, Aufklärung au sens où l'entend Kant:

Aufklärung ist der Ausgang des Menschen aus seiner selbst verschuldeten Unmündigkeit. ${ }^{8}$

Faublas par le mariage subsumant 'les amours' libertines dans L'Amour romantique, Louvet par la décision de soumettre la parole frivole du romancier et les propos éphémères du journaliste à la discipline du Tribun défendant la Liberté, croyaient sortir de la 'minorité' (Unmündigkeit) qui jusqu'alors, par leur propre 'faute' (selbst verschuldeten), avait été la leur. C'est justement alors que les héros basculent dans le malheur. Faut-il voir là un effet 'théologique' analogue à la chute d'Adam et Eve ou au châtiment de Prométhée? Peut-on y déceler une manifestation du problème fondamental de toute pensée critique de la 'raison', de Kant à Sartre? Doit-on simplement noter la concomitance de l'utopie déjà presque atteinte et d'une folie hubrique? Sans prétendre offrir de solution, puisque, comme Kant le notait prudemment nous ne sommes pas éclairés mais tentons seulement de nous éclairer, ${ }^{9}$ je vais en rapprochant certains aspects du texte romanesque et de la vie de son auteur examiner la tension pathétique entre passion(s) et raison, entre irresponsabilité et lumières, entre libertinage et liberté, et son retournement ironique dans les rapports de l'utopie et de la folie. Les trois aspects auxquels je m'arrêterai concernent la situation du sujet et sa traduction dans ses conduites, le rôle des femmes et le jeu générique entre les amours (féminin pluriel) et l'Amour (masculin singulier), 
enfin les modalités de l'énonciation, privée ou publique, romanesque ou civique, tendant vers un degré zéro ou 'passionnelle,' apologétique, dénonciatrice, pathétique.

Au moment où ils touchent à la consécration, précédant de peu celui où ils vont être la proie du malheur, Faublas comme Louvet ont certes connu des revers et même reçu des 'avertissements du destin.' Cependant rien n'a pu les arrêter, portés qu'ils étaient par leur 'étoile' dans la 'sérendipité' (dirait Walpole) des amours comblées ou de l'écriture accueillie avec faveur.

La désinvolture du Chevalier, la confiance du jeune commis de librairie tiré des 'basses lumières' (comme Brissot 'de Warville' étudié par Darnton) et devenu personnage en vue, peuvent laisser croire que rien ne leur est interdit: jusqu'ici chaque aventure leur a été utile en même temps qu'agréable.

Mais il ne faudrait pas croire que l'univers du Faublas soit celui d'un roman d'éducation avec une 'sauce' libertine. ${ }^{10}$ Le Chevalier a trop de dons naturels pour avoir besoin de l'école de l'expérience; comme les gens 'bien nés' il sait (presque) tout sans avoir rien appris, et est capable avec grâce et comme par grâce de danser - en cavalier et en 'dame,' sous le travesti -, de toucher du clavecin, de monter à cheval, de faire des armes, de converser et même de philosopher; il lui suffit d'avoir l'occasion de manifester ses qualités qui semblent découler de sa qualité, et tous ceux (ou presque), toutes celles surtout qu'il rencontre semblent se disputer le plaisir de lui offrir sa chance. S'il séduit, c'est spontanément, lorsque les conditions s'y prêtent, quand ses réactions sont 'catalysées:' son initiation sexuelle établit un schème - jeune homme imberbe, travesti pour l'amusement d'un roué, il accède au boudoir d'une grand dame libertine - est-ce sa faute si la nature reprend ses droits quand l'artifice a prétendu la mettre dans son jeu? ${ }^{11}$ Chez lui le 'libertinage' est (presque) 'ingénu.' Si la conscience de son infidélité l'amène à s'interroger sur ses infractions au code chevaleresque, il se rassure en pensant au caractère 'altruiste' de ses actions:

O ma Sophie! tu m'es bien chère; mais faut-il à cause de toi laisser la marquise mourir de chagrin? (p. 594)

Sans doute cette casuistique peut-elle sembler furtive et étriquée. Mais la belle santé de Faublas lui permet de mener de front plusieurs intrigues - divisant il est vrai les sens et la tête dominés par Mme de $\mathrm{B}^{* * *}$, 'maman' éducatrice, du coeur sur lequel règne la couventine Sophie - et même d'accorder en passant ses vigoureux hommages à la suivante ou à la demoiselle d'opéra. Les intéressées en apprenant 
les frasques du chevalier les pardonnent aisément, pour peu qu'elles aient l'occasion de faire de lui leur obligé.

Louvet de même, en 1787, a 'reçu' licence de fronder les institutions et les gens en place, en conservant les bonnes grâces de la cour; il peut être un romancier léger et un notable ayant des biens au soleil, mieux, devenir un notable, acquérir ces biens grâce au succès de son libertinage.

Pourtant les mises en garde n'ont pas manqué à Faublas ni sans doute à son créateur. Quand elle perce à jour les intrigues du chevalier, Madame de $B^{\star \star *}$, sa maîtresse en libertinage, lui fait la leçon avec bon sens:

Monsieur, votre ancien travestissement a nécessité des travestissements nouveaux, mille imprudences ont suivi la première. Quelques précautions nous ont sauvés jusqu'à présent; mais on ne saurait tromper longtemps le public curieux et malin. Le hasard qui nous a servis pourra nous perdre ...

(Une année, p. 663)

Elle met en évidence l'immaturité (Unmundigkeit) au sens mondain dont son amant et elle-même ont fait preuve. Mais il y a plus que de l'imprudence dans la légèreté de Faublas et de son créateur (qui pourtant a déjà articulé la relation du libertinage avec la liberté): il y a un défi à l'histoire. Un avertissement a été présenté en abîme, dans le récit - médiocrement documenté et sentimental mais au goût du jour en 1787 - du Comte Lovzinski:

Mon histoire offre un exemple effrayant des vicissitudes de la fortune ... Unique rejeton d'une famille illustre, ... je devais occuper dans mon pays les premières charges de $l^{\prime}$ Etat et je me vois condamné à languir à jamais sous un ciel étranger, dans une oisive obscurité ...

(Une année, p. 480)

Les maheurs du noble Polonais (qui vit caché sous un nom d'emprunt) annoncent et structurent ceux de Faublas qui écrit au terme du roman:

... J'ai perdu ma patrie et je ne puis me charger d'aucun emploi dans les armées de la république (i.e. respublica de la Pologne); il me faut, pour toute ma vie peut être, renoncer à l'état auquel je semblais appelé ...

(dernière page de la Fin des amours, p. 1222)

et de Louvet, notant en 1794 dans ses Mémoires

Qui m'eût dit, en 86, lorsque j'écrivais ( ... ) toutes les nobles infortunes de Pulawski (sic) que bientôt ma destinée auroit avec la sienne tant de frappans rapports ... ${ }^{12}$ 
Infidèle malgré lui à un serment donné à l'amitié, patriote farouche pourchassé comme rebelle, guerrier courageux réduit à faire figure de dilettante, Lovzinski/Pulaski est incapable de se justifier autrement que par la confidence, traduite par ses émules malgré eux en correspondance ou en mémoires apologétiques. Comme eux, il est victime d'apparences trompeuses qui le poursuivent alors même qu'il s'est converti à la défense de la vertu. Il a aussi été atteint dans ses affections les plus chères, par la mort de son épouse et inspiratrice, Lodoïska, et la disparition de sa fille Dorlinska (laquelle, nous l'apprendrons par la suite, n'est autre que la Sophie de Faublas, ce qui l'amènera à 'croiser' (au sens de Calvino) et à traverser le destin du chevalier par une série d'épreuves). De ce point de vue Faublas et Louvet seront moins malheureux. 'Sophie ... reste' au premier en dépit d'autres deuils. Quant au second, très affecté par l'exécution de Mme Roland, il sera soutenu par l'amour de 'sa' Lödoïska.

Si Pulaski/Lovzinski, irréprochable moralement, souffre de persécutions si exemplaires, comment Faublas qui a vécu dans l'amoralité en exploitant le 'semi-licite' des occasions offertes, comment Louvet qui s'est jeté dans la politique partisane, pourraient-ils se croire protégés des coups du sort? Ni l'un ni l'autre n'est en effet prêt à accepter la logique du pire ou l'inversion des valeurs qui permettraient de défier la fortune comme le font les personnages sadiens. Faublas et son créateur sont 'en principe' portés au bien; mais leurs actions ne réussissent pas à s'inscrire dans 'l'universel de l'entendement' kantien ('Toute action doit être telle qu'elle puisse en tous temps constituer une règle universelle') et restent dans la zone où le 'développement des lumières' (Aufklärung) se fait mal, en dépit de l'entreprise même que constitue la rédaction du roman pour Louvet. ${ }^{13}$ Avec le malheur cependant le chevalier et l'écrivain, même s'ils retombent dans les errements de leur première jeunesse, prennent conscience des tensions entre leurs désirs et le réel. Ainsi Faublas peut-il corriger la vision utopique de la charmante petite comtesse de Lignolle pour qui une chaumière et un coeur suffiraient à recréer l'univers. ${ }^{14}$ Dans le cas du Citoyen Louvet, qui se présente dans les Mémoires en 'apologiste' de son rôle de Cassandre ('Hélas, je criois dans le désert; les conspirateurs étouffoient ma voix ... ${ }^{15}$ et mes amis écoutoient sans entendre'), c'est dans le domaine des lumières mêmes que s'établit la tension, mais la moralité conquise demeure aveugle. Comme Faublas, il est 'paralysé par des serments contradictoires' comme l'écrit Charles Mervaud. ${ }^{16}$

Ce dernier poursuit: 'incapable de répondre de ses actes, le 'jeune homme aux cinquante noms' vit sous tutelle féminine ...' Il met ainsi en évidence l'effacement du sujet devant la crise, effacement qui affecte aussi Louvet en fuite et dépendant de protections féminines 
(dévouement de 'Lodoïska,' 'couverture' offerte par 'les jupes secourables' de voyageuses etc. ${ }^{17}$ ). Il me semble donc un peu rapide de parler à propos de Faublas d"épopée phallique' ou de considérer Louvet, avec son personnage, comme 'le dernier des libertins. ${ }^{18}$

La richesse du roman, l'intérêt de la vie de Louvet tiennent en effet pour une large part à la façon dont s'articulent libertinage et liberté, et à la façon dont les femmes perçoivent cette articulation. En effet, les trois principales partenaires de Faublas réussissent à surmonter 'dialectiquement' les épreuves devant lesquelles le jeune homme se trouve désemparé, assumant la responsabilité de leur destin et modelant celui du sujet masculin. Dans les textes où Louvet évoque des événements historiques, la place des femmes est également éminente, que ce soit lors des journées des 5 et 6 octobre 1789 - qui ont suscité son premier pamphlet politique - ${ }^{19}$ dans les discussions qui ont cristallisé l'idéologie 'girondine' ou à propos de l'assassinat de Marat. Le mémorialiste ne manque jamais une occasion d'exalter le courage et la vertu d'héroïnes qui montrent l'exemple aux hommes. ${ }^{20}$ Son hommage à 'Lodoïska' n'est pas une idéalisation mystifiante mais l'expression sincère de son respect et de sa gratitude, en dépit du ton romanesque qu'il adopte. Fauchery n'hésite pas à écrire non seulement que le romancier, avec la comtesse de Lignolle, nous offre

l'image la plus complète des charmes, des faiblesses et des vertus cachées de (la) femme enfant ... qui du caprice gracieux s'élève si légèrement jusqu'au sublime

mais qu'il a aussi campé avec Mme de $B^{\star \star *}$ le personnage le plus achevé de Machtfrau - en qui se cristallisent toutes les 'supériorités' de la 'femme forte' - que connaisse le roman français:

Aucune pusillanimité comme aucune mauvaise foi ne vient ternir sa liberté ... Elle chevauche comme une duchesse de Chevreuse s'habille volontiers en homme et de manière à faire illusion; pourtant elle préserve toutes les grâces de la femme jusqu'au don des larmes. C'est un Fantomas femelle ... ${ }^{21}$

Cependant l'éminent comparatiste n'accorde que peu d'attention à Sophie-Dorliska. Peut-être le caractère de l'épouse vertueuse et sensible qui n'a pas la couleur, le brio, l'audace de la marquise, 'femme faite' et mondaine, ni l'intéressante naïveté de la petite comtesse, enfant réfugiée dans un rêve utopique, est-il moins spectaculaire, et moins susceptible de fonder un paradigme. Mais c'est le développement de Sophie qui révèle le mieux les tensions qui établissent l'unité de l'ensemble romanesque, qui accompagne le mieux (de façon complémentaire 
puisqu'elle 'grandit' alors qu'il s'efface) la carrière de Faublas, qui pose le mieux la problématique de l'accès à la liberté et de la limite du libertinage, bref celle de l'Aufklärung amoureuse, le passage des 'amours' à l'Amour.

Certes on pourrait penser qu'elle ne se trouve que par défaut placée au centre du dispositif féminin. Comme le souligne Mervaud, ${ }^{22}$

Le destin choisit pour (Faublas), dénouant la situation par la mort violente de ses deux maîtresses: l'une se suicide dans une scène de roman noir, l'autre reçoit le coup d'épée destiné à son amant.

Il n'en reste pas moins que cette double catastrophe, si elle boucle l'intrigue (les intrigues), ne marque pas la fin des amours de Faublas. Si elle est efficace dramatiquement (ou mélodramatiquement) et permet d'offrir une coda 'philosophique' avec les dernières paroles de la Marquise ('Puisse du moins mon exemple avertir la foule des infortunées menacées d'un destin pareil') et une chute romantique avec celles de la petite comtesse qui va se jeter à la Seine (p. 1202), la 'double catastrophe' laisse Faublas aux prises avec les implications sociales et morales de son libertinage, avec la téléologie des amours. L'impuissance du 'héros' devant ces 'coups mortels' (p. 1204) qui le frappent en même temps, et surtout devant l'irruption brutale de la lumière dans sa conscience, l'amène à 'une frénétique évacuation du moi $^{23}$ qui se traduit par la folie. C'est alors que, tandis que ses rivales ont disparu, - la marquise dans une aura héroïque en affirmant son autonomie aristocratique par le refus de renier son engagement amoureux libertin tout en en déplorant les conséquences néfastes, la comtesse dans un désespoir ophélique qui la conduit à rechercher un retour solitaire à l'harmonie, dans une nostalgie utopique, solution passionnelle à l'amour impossible - l'épouse légitime entreprend de sauver de Faublas et de l'Amour ce qui peut raisonnablement être sauvé, ici, maintenant, dans le réel. Elle va exorciser les fantasmes culpabilisants du Chevalier, dans un mimodrame projectif (mis en scène par l'aliéniste Willis, ${ }^{24}$ et auquel participent le père et la soeur de Faublas). Ce traitement, qui appellerait une analyse détaillée, part de la confusion dans l'esprit du malade entre ses proches, présents devant lui, et les personnages disparus qui le hantent (pp. 1206-07); il va peu à peu, par une série de tableaux qui rappellent le patient à la réalité de sa perte, l'amener à reconnaître la réalité de ce qui 'lui reste.' Le rôle principal y est dévolu à Sophie, chargée chaque soir de prononcer l'anti-sentence susceptible de conduire à la (re)conquête de la responsabilité (Aufklärung):

Pourquoi l'arrêter? Qu'on ouvre toutes les portes! Qu'il soit libre! 
Mais c'est l'initiative de la jeune femme qui modifie le scénario de façon décisive.

Ce soir elle crie 'Qu'il soit libre!' et soudain elle s'échappe, elle se précipite, elle arrive avant son époux au bosquet dont elle lui défend l'entrée. 'Que venezvous chercher?' lui dit-elle. Sans la regarder il répond: 'Je cherche un tombeau' ... . 'Pourquoi chercher un tombeau, mon bien-aimé? Ta Sophie n'est pas morte.' Il s'écrie: 'C'est la voix secourable!' et levant les yeux sur elle: 'Sophie! ... dieux! ma Sophie!'

(p. 1211: '16 mai 1785')

Le docteur accouru peut justement commenter:

L'amour heureusement téméraire a commencé la guérison: que l'amour l'accomplisse et qu'il y soit aidé par la nature ...

avant d'organiser un tableau 'synoptique' de tout ce qui attache Faublas au réel et peut l'arracher à son 'rêve affreux,' perçu comme constitué d'atroces souvenirs.

En catalysant la reconnaissance, en situant la liberté dans le présent sensible, en assumant la responsabilité d'une relation avec l'Autre aliéné, Sophie actualise l'Amour et dissipe les sortilèges des amours. Elle rend à son époux la faculté d'agir et d'expier les conséquences de ses égarements. Mais Faublas, sauvé et puni, est dépouillé de son infinie disponibilité de libertin, et doit accepter l'exil loin de la France et s'engager à ne pas servir d'autre pays. Sa liberté, circonscrite et contingente, est indissociable de celle de Sophie, puisqu'il abandonne les 'tombeaux' de ses maîtresses pour vivre dans le château que sa femme a recouvré en Pologne. Il se trouve dans la situation initiale de Sophie, jeune exilée qui découvrait l'amour dans une chasteté contingente. ${ }^{25}$ Son assimilation aux femmes qui dans la première partie du roman s'effectuait par le travesti (dont l'étude serait d'un extraordinaire intérêt, avec ses incidences sur la représentation du moi, les jeux de l'identité et des rôles sexuels, la tonalité quelque peu narcissique du libertinage) devient ici politique: il se retrouve homme au foyer et 'citoyen passif,' situation qui préfigure à bien des égards celle de Louvet à la fin de sa vie.

Je ne puis qu'esquisser la problématique de l'énonciation dans le texte romanesque et dans les publications politiques de Louvet. ${ }^{26}$ Dans les unes comme dans l'autre une dichotomie est perceptible entre la prise de parole et et la perte de parole: Faublas, dans sa démence, est pris en charge par ses proches, et c'est son père qui raconte son traitement; Louvet 'interdit de publication' devra recourir à l'auto-édition pour se 
faire entendre dans la sociéte thermidorienne réactionnaire. Pour l'un comme pour l'autre l'expression aisée du début est associée à la libre disposition de l'argent. Dans les deux cas également l'énoncé est 'mixte' ou plutôt complexe. Le récit des amours est coupé de réflexions, de récits enchâssés (histoire de Lovzinzki par exemple), de scènes dramatiques présentées comme de véritables dialogues de théâtre insérés (pp. 1118-28, 1131-39) ou graduellement actualisés (pp. 1008-09, 1010-16), de rapports, de discours, de lettres programmatiques, (celles de Lovzinzki au père du Chevalier éclairent les desseins du beau-père persécuteur devenu ainsi maître-initiateur (pp. 953-60, 1195)); ce texte fictif fait d'ailleurs appel à des effets de réel intéressants qui font de Faublas un vaste roman de moeurs. Quant aux discours du député, ils comportent des éléments de 'politique fiction' comme le notait Aulard, les Mémoires combinant témoignage sur les événements publics, relation, souvenirs personnels et tentative de justification (apologia).

Cependant une différence importante semble séparer les énoncés donnés comme fictifs (romans de $\mathrm{M}$. Louvet de Couvray, aristocrate lui-même fictif, comme il le signale plaisamment dans le texte préfaciel 'à mon sosie' (pp. 415-16), auquel il ajoute en note désinvolte en 1790 'la plus impertinente des révolutions m'enlève ma noblesse d'hier. Que je suis heureux d'avoir un nom de baptême!') des propos du Citoyen Louvet. Le roman débute 'en moi' et ce n'est qu'au moment de la folie, où le personnage devient objet, que domine la troisième personne, Faublas guéri reprenant la parole dans les toutes dernières lettres, datées de l'exil. Les textes politiques en apparence plus objectifs sont 'en fait plus rusés encore,' et font appel simultanément à des ressources rhétoriques variées et complexes. Ainsi, la 'Robespierride,' morceau hanté par les Catilinaires, décrit d'abord le chef montagnard de l'extérieur ('On vit alors un homme' ... ); mais la parole se fait bientôt passionnée, directe, ad hominen, non pour atteindre un sujet, mais pour déconstruire un objet:

Je t'accuse de t'être perpétuellement produit comme un objet d'idôlatrie, d'avoir souffert que devant toi on te désignât comme seul homme vertueux ...

Or la mise en cause de l'histrion qu'est 'l'incorruptible' joue sur des effets histrioniques, l'invocation à la vraie vertu pour dénoncer la 'fausse' repose sur une dérive illusoire: 'Je t'accuse enfin d'avoir évidemment marché au suprême pouvoir.' La 'transparence' du justicier est artificieuse et ironiquement piégée. ${ }^{27}$ Les Mémoires eux aussi font appel à mille ressources impures pour défendre une cause 'pure' et 'vraie.' Les accusations contre Robespierre s'articulent par exemple sur des ragots diffamatoires: 
un Monsieur Nicolas ... dont Camille Desmoulins parle dans son Vieux Cordelier ... doit sa petite fortune ... et le droit de vie et de mort qu'il exerce contre tous les gens de bien, au gros bâton dont il rassura la lâcheté naturelle de Monsieur Robespierre. ${ }^{28}$

Tout se passe donc comme si la Terreur avait contaminé ses victimes et comme si 'l'intoxication' de l'esprit public que Louvet attribue à ses persécuteurs contraignait le fugitif à mentir à son tour, dans l'exposé même de la 'vérité.' Parmi les crimes que l'on commet 'au nom de la liberté,' le terrorisme de l'expression est peut-être un des plus insidieux.

La persécution subie par Louvet a donc des effets voisins de ceux que les malheurs exercent sur Faublas. Mais le délire de l'écrivain est conscient, et la crise salutaire de Thermidor ne réussit pas à le délivrer.

\section{Conclusion}

Roman léger, Faublas? A qui peut-on le faire croire, si l'on en présente le texte complet et si l'on en combine la lecture avec l'histoire sur la trame de laquelle il se projette et que Louvet aura le malheur de vivre. Les amours en cascade du chevalier masquent un vrai péril, et l'histoire en abîme du comte (j'allais dire du commandeur) Lovzinzki dans la Pologne du premier partage dessine celle du libertin qui fait maladroitement l'apprentissage des lumières, et celle du romancier qui tente le passage des fictions privées à la vie publique dans la France où la liberté bute sur la terreur. Certes Faublas au terme du roman trouve un asile étranger (pour lui) dans la République de Pologne de 1786. On sait la précarité de cet asile. De même Louvet, échappé à la guillotine, regagnera Paris, ville jouisseuse, affairiste, 'étrangère' et mourra sans avoir vu la chute de la République Une et Indivisible. Livre et carrière du romancier se construisent ainsi pour les sujets masculins fragmentés en sursis sur les chemins de la liberté et de l'Aufklärung. Mais, et l'ironie ici est positive, les femmes qui se projettent dans ce livre (autrement que par l'effet narcissique du travesti), ou se manifestent dans cette carrière, réussissent à transcender le libertinage par la mort héroïque, par l'utopie, par le dévouement et le réalisme amoureux qui les constituent en sujets unifiés, à part entière et librement éclairés.

Pour Faublas et son créateur, le deuxième sexe, celui que son orgueil et sa 'force' illusoire risquent de faire basculer dans l'altérité de la folie, est-il masculin?

PIERRE GOBIN

Queen's University 


\section{Notes}

1 Romanciers du XVIIIe siècle II, Texte (pp. 403-1222) et notes (pp. 1968-91) par Marguerite du Cheyron et Etiemble, (Paris: N. R. F. Gallimard (Bibliothèque de la Pléiade) 1965.)

2 La particule est trompeuse: le Couvray n'est en fait que le nom d'une terre. F. A. Aulard a réédité en 1889 avec une préface, les Mémoires de Louvet, ainsi que le texte du célèbre discours du 29 octobre 1792 A Maximilien Robespierre et à ses royalistes. Aulard a utilisé ces textes ainsi que les cinq volumes de la Sentinelle, journal républicain dirigé par Louvet entre 1795 et 1797 dans son Histoire politique de la Révolution Française, édition définitive de 1903 (Paris, A. Colin). Je cite les Mémoires d'après l'édition originale intitulée 'Quelques notices pour l'histoire et le récit de mes périls depuis le 31 mai 1793,' Paris, l'an IIIe de la République. De larges extraits de 'la Robespierride' figurent dans l'ouvrage de Bernardine Melchior-Bonnet, les Girondins (Paris: Perrin 1969), pp. 172-76.

3 Si son destin 'encadre' celui des Girondins, ses positions sont assez originales: hostile à l'exécution du roi 'tant que la constitution ne sera (it) pas en vigueur,' partisan de la paix alors que ses amis se montraient bellicistes, adversaire résolu de Robespierre, tandis que la plupart s'acharnaient sur Marat puis Danton. Taxé de 'Fédéralisme' en l'an II, pourchassé comme 'droitier' durant la Terreur jacobine, il ne dirigera pas moins l'un des quatre organes de presse demeurés 'franchement républicains' sous le Directoire. Louvet apparaît comme un 'homme à principes,' champion des causes impopulaires.

4 Histoire p. 140: 'Il ne s'apercevait même pas, quand il prêtait aux Montagnards de compliqués desseins royalistes, qu'il était la dupe de son imagination ... il prenait ses fables pour la réalité.'

5 Melchior-Bonnet Les Girondins épilogue (p. 482), relate les dernières années de la vie de Louvet qui 'ne furent pas heureuses,' les persécutions dont sa femme et lui furent l'objet de la part des Muscadins royalistes, ses démêlés avec la presse et les libraires (procès en diffamation, édition pirate de Faublas), le délabrement de sa santé et sa mort prématurée ('le 24 août 1797 ... une phtisie galopante l'emporta. Il n'avait que trente-sept ans.')

6 Cf. les Mémoires de Louvet lui-même sur sa fuite; Aulard (Histoire politique) pp. $568-69$ et 509 .

7 'Les Girondins se levèrent avec la Montagne pour m'empêcher de parler' note Louvet avec amertume dans ses Mémoires. Camille Desmoulins persifle l'auteur de la 'Robespierride,' et compare l'Incorruptible avec 'Hercule aux prises avec un basset enragé' (Melchior-Bonnet p. 180).

8 'Beantwortung der Frage: Was ist Aufklärung?' 1784 (in Kant's Gesammelte Schriften vol. VIII, Académie Royale de Prusse, Berlin, Reimer 1912), pp. 33-42. La citation est la phrase initiale. Je n'ai pu déterminer si Louvet avait eu connaissance de l'essai du Philosophe de Königsberg.

9 ibid p. 40. 'Wenn denn nun gefragt wird: Leben wir jetzt in einem aufgeklärten Zeitalter? So ist die Antwort: Nein, aber wohl in einem Zeitalter der Aufklärung.'

10 Il y aurait lieu toutefois d'examiner le rôle de Lovzinzki dans La Fin: il soumet Faublas à une série de tests initiatiques qui pourraient, comme ceux de La Flûte enchantée, avoir un corrélat maçonnique. 
11 Il n'y a donc rien en lui qui rappelle la diabolie étudiée par Claude Reichler (Paris, ed. de Minuit, 1979).

12 Mémoires, p. 13. On notera que l'histoire des 'Pulawski,' plus ou moins authentique mais télescopée, est elle-même caution de celle de Lovzinzki. (Elle sert de 'texte tuteur' au sens de Barthes.)

13 Mémoires, pp. 152-53.

14 La Fin ... p. 1113. La description du 'statut' des amants définit ce que Kant appelle Unmundigkeit (cf note 9 ci-dessus).

15 Mémoires, p. 30, sur l'époque 'à jamais fatale du 10 mars 1793.'

16 Article 'Louvet, de Couvray Jean Baptiste' dans le Dictionnaire des littératures de langue française, Paris, Bordas 1984 (sous la direction de J. P. de Beaumarchais, Daniel Couty, Alain Rey), vol. II, pp. 1337-39.

17 Mémoires, p. 135.

18 Fauchery, la Destinée féminine dans le roman ... (Paris: Colin, 1972), p. 65, qui pourtant a si bien analysé les personnages féminins; $\mathrm{M}$. Crouzet préface à l'édition, pp. 10-18.

19 Paris justifié contre M. Mounier. Paris, Bailly, 1789. Dans les Mémoires (p. 3) Louvet attribue à la 'faction d'Orléans' (l'entourage du futur Philippe-Egalité) la responsabilité des forfaits qui ont 'souillé ... ces journées' conduites par les femmes du peuple réclamant du pain.

20 Cf. dans les Mémoires (p. 152) le récit de l'exécution de Mme Roland qui console l'homme qui va monter après elle à l'échafaud.

21 Fauchery, p. 530 (Mme de L); p. 615 (Mme de B).

22 Dictionnaire, pp. 1337-39.

23 ibid.

24 La Fin Pléiade pp. 1207-15. Le récit du traitement est fait par le père du chevalier. Le docteur Willis, personnage réel, a traité (sinon 'guéri' comme le suggère la note des éditeurs p. 1986) le roi George III. Le traitement relève de la réalisation théâtrale selon Michel Foucault (cité à la note id, ibid).

25 Certes, elle était une couventine impubère en deçà du libertinage, alors que lui se trouve au-delà.

26 Une telle étude ferait intervenir l'autre roman de Louvet Emilie de Varmont ou le divorce nécessaire et les amours du curé Sevin, Paris, Bailly 1791, ainsi que les articles de la Sentinelle.

27 Aulard (Histoire politique ... , loc. cit.); Nagy, Peter (Libertinage et révolution, Paris Gallimard, 'idées', 1966); on pense à l'analyse de Claude Reichler op cit (pp. 106-110) oú il discute le réquisitoire perverti du cerf Brichemer (cf italien briccone) dans le Roman de Renart.

28 Mémoires, p. 31. Cet affrontement 'terroriste' (après la Terreur) des 'derniers libertins' serait à lire à la lumière des travaux de Lefort et de Poulantzas. 